\title{
Liposomal Formulation Decreases Toxicity of Amphotericin B In Vitro and In Vivo
}

\author{
Justin Roberts MD, Josh Bingham MD, \\ Alex C. McLaren MD, Ryan McLemore PhD
}

Published online: 25 March 2015

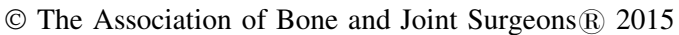

\begin{abstract}
Background Liposomal amphotericin B is locally delivered to treat fungal orthopaedic infections but little is known about local tissue toxicity, if any, that might be associated with local delivery.

Questions/purposes (1) Is liposomal amphotericin B cytotoxic in vitro? (2) Is locally delivered liposomal amphotericin B toxic to tissue in vivo?

Methods Mouse fibroblasts (BA LB/3T3 A31) and osteoblasts (MC3T3) were exposed to two formulations of amphotericin B (liposomal and deoxycholate) at concentrations of $0,1,5,10,100,500$, and $1000 \mu \mathrm{g} / \mathrm{mL}$. Cell viability was determined by MTT assay after 1,3 , and 5 hours of exposure and a proliferation assay after 1, 4, and 7 days of exposure and then after 3 recovery days without drug. Tissue exposure occurred by local delivery of liposomal amphotericin B, 200 or $800 \mathrm{mg} /$ batch antifungalloaded bone cement (ALBC), or amphotericin B deoxycholate, $800 \mathrm{mg} / \mathrm{batch}$ ALBC in rat paraspinal muscles.
\end{abstract}

One or more of the authors (ACM, RM) was supported by a grant of more than USD 10,000 from Astellas Scientific and Medical Affairs, Inc (Northbrook, IL, USA).

All ICMJE Conflict of Interest Forms for authors and Clinical Orthopaedics and Related Research ${ }^{\mathbb{B}}$ editors and board members are on file with the publication and can be viewed on request.

Clinical Orthopaedics and Related Research ${ }^{\circledR}$ neither advocates nor endorses the use of any treatment, drug, or device. Readers are encouraged to always seek additional information, including FDAapproval status, of any drug or device prior to clinical use.

Each author certifies that his or her institution approved the animal protocol for this investigation and that all investigations were conducted in conformity with ethical principles of research.

J. Roberts, J. Bingham, A. C. McLaren ( $\varangle)$, R. McLemore Orthopaedic Department, Banner Good Samaritan Medical Center, 1320 N 10th Street, Phoenix, AZ 85006, USA

e-mail: amclaren@cox.net
White blood cell count (WBC) and serum amphotericin B levels were obtained on Days 1 and 3. Rats were euthanized at 2 and 4 weeks and semiqualitative histopathology was performed.

Results Liposomal amphotericin B is cytotoxic in vitro but not toxic to tissues in vivo. All cells survived concentrations up to $1000 \mu \mathrm{g} / \mathrm{mL}$ for 5 hours, $100 \% \pm 0 \%$, but none survived $\geq 100 \mu \mathrm{g} / \mathrm{mL}$ for 7 days, $0 \% \pm 0 \%$. Fibrosis was seen adjacent to ALBC without inflammation or necrosis, indistinguishable from controls for both liposomal amphotericin B doses. Amphotericin B serum levels were all less than $1 \mu \mathrm{g} / \mathrm{mL}$ and $\mathrm{WBC}$ counts were all normal.

Conclusions In vitro cytotoxicity to liposomal amphotericin B occurred but no adverse tissue reaction was seen in vivo.

Clinical Relevance Local delivery of liposomal amphotericin B in ALBC was well tolerated by mouse tissue; however, clinical studies are needed to confirm this finding in humans.

\section{Introduction}

Orthopaedic infections caused by fungi are highly resistant to antifungals as a result of the formation of fungal biofilms [10]; thus, high antifungal concentrations are required for treatment. The mean biofilm-eliminating concentration (MBEC) for fungi in biofilm can be hundreds of times higher than the minimum inhibitory concentration $(0.030-4.0 \mu \mathrm{g} /$ $\mathrm{mL}$ ) for the same fungi not in a biofilm [13]. Amphotericin B is a commonly used antifungal that has severe systemic toxicity limiting the daily dose to $1.5 \mathrm{mg} / \mathrm{kg} /$ day and infusion concentration to $100 \mu \mathrm{g} / \mathrm{mL}$. To prevent systemic toxicity, infusion of $50 \mathrm{mg}$ generally takes 4 to 6 hours, leading to serum levels of $2 \mu \mathrm{g} / \mathrm{mL}$ or less [5]. MBEC of 
amphotericin B for fungi known to cause orthopaedic infections cannot be achieved safely by parenteral administration. Local delivery has been proposed to achieve MBEC using amphotericin B deoxycholate in antifungalloaded bone cement (ALBC) as an adjuvant to surgical débridement [11]. We have previously studied ALBC with amphotericin B deoxycholate [8], finding limited release and significant toxicity to fibroblasts and osteoblasts at relatively low concentrations [6]. We subsequently showed that both liposomal amphotericin B [4] and voriconazole [12] are delivered from ALBC in much greater amounts than amphotericin B deoxycholate. Liposomal amphotericin B is known to be less toxic than amphotericin $\mathrm{B}$ deoxycholate when delivered intravenously [2]; however, there are no reported data on the local toxicity from locally delivered liposomal amphotericin B. We therefore asked: (1) Is liposomal amphotericin B toxic to fibroblasts and osteoblasts in vitro? (2) Is locally delivered liposomal amphotericin B toxic to tissue in vivo?

\section{Materials and Methods}

To determine in vitro cellular toxicity from liposomal amphotericin B, mouse fibroblasts and osteoblasts were exposed to serial concentrations of liposomal amphotericin B (Ambisome; Astellas US LLC, Northbrook, IL, USA), or amphotericin B deoxycholate, for up to 7 days. Cell viability was assayed either with MTT proliferation assay (Promega. Madison, WI, USA) for 5 hours or less or assayed with alamarBlue ${ }^{\circledR}$ (Life Technologies, Thermo Fischer Scientific, Carlsbad, CA, USA) for 1 to 7 days. To determine in vivo tissue toxicity from liposomal amphotericin B, ALBC was implanted in the paraspinal muscles of Sprague-Dawley rats (Charles River Laboratories, Wilmington, MA, USA). Rats were euthanized at either 2 or 4 weeks for histologic evaluation. Blood was collected for serum amphotericin B levels as an indicator for systemic exposure and white blood cell (WBC) count as measured as an indicator of systemic response.

\section{In Vitro Toxicity}

To determine cellular toxicity, mouse fibroblasts (BA LB/ 3T3 A31; American Type Culture Collection; Manassas, VA, USA) and mouse osteoblasts (MC3T3; American Type Culture Collection) were grown in Dulbecco's Modified Eagle's Medium with $2 \mathrm{mmoL}$ glutamine/L, 5\% bovine serum, $10 \mathrm{U}$ penicillin/mL, and $10 \mu \mathrm{g} / \mathrm{mL}$ streptomycin (all from Fisher Scientific, Pittsburgh, PA, USA). The cells were plated in a 96-well plates with 20,000 cells per well and then exposed to concentrations of $0,1,10,100,500$, and $1000 \mu \mathrm{g} /$ $\mathrm{mL}$ of either of two amphotericin B formulations: liposomal amphotericin B as the experimental group and amphotericin B deoxycholate for comparison. Negative controls with no drug were also performed $(n=6$ per each drug/concentration/time/control group). After 1, 3, and 5 hours of exposure to drug, an MTT proliferation assay was performed to determine the presence of live cells. Absorbance was measured on a FLUOSTAR Omega Multiplate Reader (BMG Labtech, Cary, NC, USA). The absorbance was normalized to the no drug group and expressed as a percent of viable cells. Cell morphology was observed under light microscopy after each time point.

Cytotoxicity and recovery from prolonged exposure to amphotericin B were determined by plating fibroblasts and osteoblasts at 500 cells per well in 24 -well plates and exposing them to concentrations of $0,1,5,10,100,500$, and $1000 \mu \mathrm{g} / \mathrm{mL}$ of either of the two amphotericin B formulations: liposomal amphotericin $\mathrm{B}$ as the experimental group and amphotericin B deoxycholate for comparison. No drug controls were also performed. At 1, 4, and 7 days, the drug-containing medium was removed and replaced with fresh medium containing alamarBlue ${ }^{\circledR}$ for 4 hours ( $n=6$ per each drug/concentration/time/control group). Fluorescence in the living cells from alamarBlue ${ }^{\circledR}$ reduction was measured on a fluorostar Omega Multiplate reader (BMG Labtec Inc, Cary, NC, USA) to detect living cells. For the samples that had amphotericin B exposures of 1 and 4 days, the medium with alamarBlue ${ }^{\circledR}$ was removed after measuring fluorescence and fresh medium with the proper concentration of each amphotericin B formulation was reapplied, allowing cells to continue to grow while exposed to amphotericin B. After the assay for 7 days of drug exposure, cells had drug-free media applied and were allowed to grow for a further 3 days. Analysis using alamarBlue ${ }^{\circledR}$ fluorescence at 10 days was performed to assess recovery that occurred over the intervening 3 days.

\section{In Vivo Response}

\section{Cement Formulation}

Three formulations of ALBC and bone cement without antifungal were made. Simplex P bone cement (Stryker, Mahwah, NJ, USA) was mixed with liposomal amphotericin B, 200 and $800 \mathrm{mg} /$ batch, or amphotericin B deoxycholate, $800 \mathrm{mg} / \mathrm{batch}$, or mixed without antifungal. The antifungal powder was homogeneously mixed with the polymer component of the bone cement and polymerized by hand-stirring with the monomer in an open bowl. ALBC rods weighing approximately $300 \mathrm{mg} / \mathrm{cm}$ were formed by 
using a 5-cc syringe to inject dough-phase ALBC into a 15French, red rubber catheter as a mold.

\section{Surgical Procedure}

All animal protocols were approved by the Institutional Animal Care and Use Committee before initiation of any animal testing. Six groups of six rats each (three for 2 weeks and three for 4 weeks; total 36) were used for the tissue toxicity investigation. Four groups had an ALBC or control rod surgically implanted in the rat paraspinal muscles: liposomal amphotericin B ALBC, either $200 \mathrm{mg}$ or $800 \mathrm{mg}$ per batch, amphotericin B deoxycholate $800 \mathrm{mg}$ per batch, or no drug (polymethylmethacrylate [PMMA] as a negative control). The remaining two groups were the positive control in which methyl cyanoacrylate (MCA) was injected in the same paraspinal intramuscular site (positive control known to cause tissue necrosis) or a sham procedure with surgical preparation of the paraspinal site but no implant or injection (Table 1). At the time of surgery, the rats were anesthetized using $2 \%$ isoflurane. A $2-\mathrm{cm}$ paramedian incision was made in the skin adjacent to the spinous processes down to the fascia. A hemostat was used to create a longitudinal cavity in the paraspinal muscles. The ALBC rod was cut at a $10-\mathrm{mm}$ length, weighed, and inserted under direct vision. Fascia, subcutaneous tissue, and skin were closed in layers.

\section{Histology Examination}

At the 2-week and 4-week time points, all three rats from each group were euthanized by $\mathrm{CO}_{2}$ asphyxiation. After euthanasia, a 5-cm skin incision was made over the original surgical site. The implant was identified by palpation, and the tissue around the implant was carefully excised, ensuring that the implant remained in the surrounding tissue without disruption. Two sutures (superficial and rostral) were placed for orientation. Specimens were placed in tissue cassettes, fixed in $10 \%$ formalin, and shipped to

Table 1. Experimental design for local amphotericin exposure

\begin{tabular}{lll}
\hline Group & 2 weeks & 4 weeks \\
\hline Amphotericin B liposomal: $200 \mathrm{mg}$ & 3 & 3 \\
Amphotericin B liposomal: $800 \mathrm{mg}$ & 3 & 3 \\
Amphotericin B deoxycholate: $800 \mathrm{mg}$ & 3 & 3 \\
No drug & 3 & 3 \\
Positive control (methyl cyanoacrylate) & 3 & 3 \\
No implant (sham operation) & 3 & 3 \\
Totals & 18 & 18 \\
& \multicolumn{2}{|c}{36} \\
\hline
\end{tabular}

Laboratory Animal Pathology Services at the University of Georgia (Athens, GA, USA) for processing. Sections from each specimen were stained with hematoxylin and eosin, Mason's trichrome, and Giemsa stain. Histologic specimens were examined independently by two blinded observers (RM, ACM) for comparison to negative controls of inflammation, lymphocytic activity, necrosis, or other cellular/tissue response based on cells per high-powered field: none (0), very slight (1-5), mild (6-15), moderate (16-25), or marked (26+) (ASTM F981-04 2010 [14]).

At Days 1 and 3, approximately $1 \mathrm{~mL}$ of blood was drawn from the tail vein using a 24-G needle for amphotericin B levels and WBC count. The WBC counts were referenced to standard ranges for Sprague-Dawley rats [9]. Blood levels of amphotericin B were measured using high-performance liquid chromatography. Samples were extracted with room temperature methanol added to the test serum (50/50 vol/ vol). Separation was performed isocratically on an Agilent 1100 HPLC (Agilent Technologies, Santa Clara, CA, USA) using a $\mathrm{C} 18$ column. Mobile phase was 58\% 10 mmol EDTA and 10 mmol sodium acetate, $\mathrm{pH} 3.6$ in water, and $42 \%$ acetonitrile at $0.8 \mathrm{~mL} / \mathrm{min}$ [7]. Detection was performed at $405 \mathrm{~nm}$. The WBC count was performed by Antech Diagnostics (Phoenix, AZ, USA). Normalized absorption data for the MTT proliferation assay and fluorescence data for alamarBlue ${ }^{\circledR}$ proliferation assay in living cell culture were compared using analysis of variance (ANOVA) with t-tests for post hoc comparisons [1]. Standard normal plots of residuals were constructed on the ANOVA results [1].

\section{Results}

\section{In Vitro Toxicity}

Liposomal amphotericin B is cytotoxic in vitro (Table 2). There was no loss of viable cells or abnormal morphology on brightfield visualization when fibroblasts and osteoblasts were exposed to concentrations of liposomal amphotericin B up to $1000 \mu \mathrm{g} / \mathrm{mL}$ for 5 hours (viability $=100 \% \pm 0 \%$, mean difference $=0[95 \%$ confidence interval $\{\mathrm{CI}\}, 100-100], \mathrm{p}=1$ ) (Table 2). However, when the exposure is 7 days, concentrations as low as $5 \mu \mathrm{g} / \mathrm{mL}$ cause a decrease in viable cells (fibroblast viability $=$ $14 \% \pm 4 \%$, mean difference $=86$ [95\% CI, 82-90], $\mathrm{p}<0.001$; osteoblast viability $=82 \% \pm 9 \%$, mean difference $=18$ [95\% CI, 9-27], $\mathrm{p}=0.004$ ) (Table 2). When compared with the deoxycholate formulation, cytotoxicity from the liposomal formulation occurred at higher concentrations ( $p<0.001$ analysis of variance) (Table 2$)$. For fibroblasts that had exposure for 7 days, $5 \mu \mathrm{g} / \mathrm{mL}$ of amphotericin B deoxycholate caused total loss of viability $(0 \% \pm 0 \%$, mean difference $=100 \quad[95 \%$ CI, 0-0], 


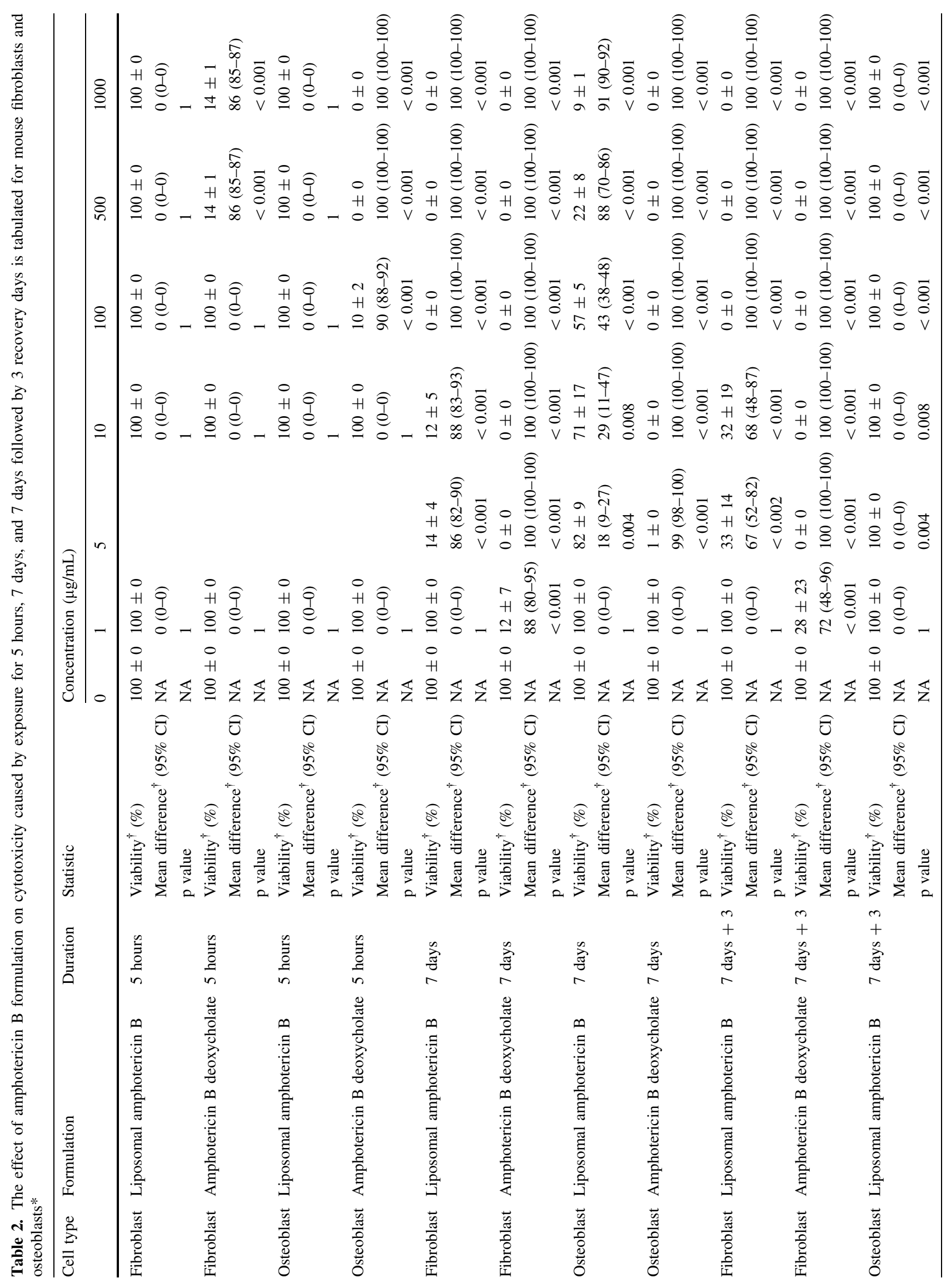




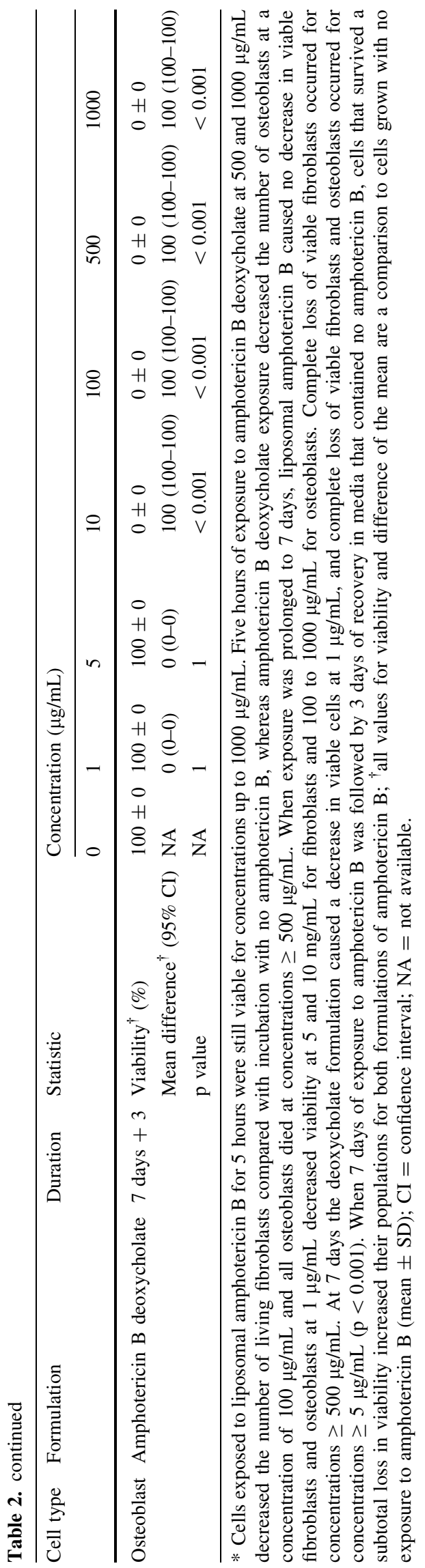

$\mathrm{p}<0.001$ ), but liposomal amphotericin B took $100 \mu \mathrm{g} / \mathrm{mL}$ to cause total loss of viability $(0 \% \pm 0 \%$, mean difference $=100[95 \%$ CI, 0-0], $p<0.001$ ). For liposomal amphotericin B, by Day 7,1 $\mathrm{g} / \mathrm{mL}$ caused no decrease in viable fibroblasts or osteoblasts (viability $=100 \% \pm 0 \%$, mean difference $=0$ [95\% CI, 100-10 0], $\mathrm{p}=0.0$ ), 5 and $10 \mu \mathrm{g} / \mathrm{mL}$ caused a decrease in viable cells (fibroblast viability at $5 \mu \mathrm{g} / \mathrm{mL}=14 \% \pm 4 \%$, mean difference $=86$ [95\% CI, 82-90], p < 0.001; fibroblast viability at $10 \mu \mathrm{g} /$ $\mathrm{mL}=12 \% \pm 5 \%$, mean difference $=88 \quad[95 \% \quad \mathrm{CI}$, 83-93], $\mathrm{p}<0.001 ;$ osteoblast viability at $5 \mu \mathrm{g} / \mathrm{mL}=$ $82 \% \pm 9 \%$, mean difference $=18 \quad[95 \%$ CI, 9-27], $\mathrm{p}=0.004 ;$ osteoblast viability at $1000 \mu \mathrm{g} / \mathrm{mL}=9 \% \pm$ $1 \%$, mean difference $=91[95 \% \mathrm{CI}, 90-92], \mathrm{p}<0.001$ ); and concentrations $\geq 100 \mu \mathrm{g} / \mathrm{mL}$ caused complete loss of fibroblast viability (viability at $100 \mu \mathrm{g} / \mathrm{mL}=0 \% \pm 0 \%$, mean difference $=100[95 \% \mathrm{CI}, 0-0], \mathrm{p}<0.001)$ and (Table 2). Amphotericin B deoxycholate at day 7 caused a decrease in viable cells at $1 \mu \mathrm{g} / \mathrm{mL}$ for fibroblasts (viability $=12 \% \pm 7 \%$, mean difference $=88 \quad[95 \% \quad \mathrm{CI}$, 80-95], $\mathrm{p}<0.001$ ) and at $5 \mu \mathrm{g} / \mathrm{mL}$ for osteoblasts (viability $=1 \% \pm 0 \%$, mean difference $=99 \quad[95 \%$ CI, 98-100], $\mathrm{p}<0.001$ ) and complete loss of both viable fibroblasts and osteoblasts for concentrations $\geq 10 \mu \mathrm{g} / \mathrm{mL}$ (viability $=0 \% \pm 0 \%$, mean difference $=100[95 \% \mathrm{CI}$, $100-100], \mathrm{p}<0.001)$. For both formulations of amphotericin $\mathrm{B}$, when a subtotal loss of viable cells occurred, surviving cells increased their numbers during the 3 recovery days after removal of the amphotericin B from the growth media (fibroblasts Day $7=13 \% \pm 4 \%$, Day $10=32 \% \pm 16 \%$, mean difference $=19$ [95\% CI, 9-29], $\mathrm{p}=0.004) ; \quad$ osteoblasts Day $7=48 \% \pm 7 \%$, Day $10=93 \% \pm 5 \%$, mean difference $=45$ [95\% CI, 38-52], $\mathrm{p}=0.027$ ) (Table 2). No recovery was seen for concentrations that caused complete loss of viable cells at Day 7.

\section{In Vivo Response}

Liposomal amphotericin B did not cause tissue toxicity in vivo for up to 4 weeks when delivered locally from ALBC made with 200 or $800 \mathrm{mg} / \mathrm{batch}$. There was mild fibrous encapsulation but none or very slight acute inflammation lymphocytic activity or other cellular response and no necrosis for all drug loads at both 2 and 4 weeks (Fig. 1B-E). The tissue response to all ALBC rods was histologically indistinguishable from the response to the no-drug rods with no disagreement between observers. The methyl cyanoacrylate group differed from all other groups with muscle necrosis, mild acute inflammation, and mild lymphocyte activity at 2 weeks and at 4 weeks (Fig. 1F).

Serum amphotericin B concentration was less than $1 \mu \mathrm{g} /$ $\mathrm{mL}$ for all animals in all groups (Table 3). The maximum 
serum concentration detected was $0.56 \mu \mathrm{g} / \mathrm{mL}$, and amphotericin B concentrations were not consistent among animals in the same treatment group. Most of the measurements were near the lower detection limit of the system. The WBC count was normal in all animals at all time points, except in a single MCA animal at 2 weeks that had a WBC count of 11.2 (upper limit of normal $=10.3 \mathrm{k} / \mathrm{mm}^{3}$ ).

\section{Discussion}

The antifungal levels needed to kill fungi in biofilms, MBEC, can be hundreds of times higher than the levels needed to kill planktonic fungi $[3,13]$. Fragments of biofilm remain in surgical sites postdébridement. ALBC is being used to achieve MBEC in postdébridement surgical sites because parenteral antifungal drugs cannot achieve levels in those ranges without risking unacceptable toxicity $[3,13]$. There is a need for safety data on antifungals in ALBC. In our study, we asked: (1) Is liposomal amphotericin B toxic to fibroblasts and osteoblasts in vitro? (2) Is locally delivered liposomal amphotericin B toxic to tissue in vivo?

There are a number of limitations to our study. First, the in vitro portion of our study is not directly applicable to clinical practice. Although both amphotericin B formulations we studied caused toxicity in cell cultures of mouse fibroblasts and osteoblasts, the concentrations that occur in vivo are unknown and it is possible that cells in living tissues are more resilient to drug toxicity. Second, nonhuman fibroblasts and osteoblasts were used. It is unknown whether human fibroblasts and osteoblasts would behave similarly. Third, we did not measure the amount of free drug in the media. It is possible that liposomal amphotericin B is less toxic because it is in liposomal form in the cell culture media, leaving less free drug to injure the cells. Fourth, alamarBlue ${ }^{\circledR}$ is an indirect indicator of cell viability. However, its sensitivity is as few as 50 cells and there is a linear response with intensity of the fluorescence to 50,000 cells. Cell growth is unaffected by alamarBlue ${ }^{\circledR}$ in the growth media. Although a few viable cells could be missed, it is an established assay for the cellular response to toxic agents and we would expect viable cells to replicate to numbers above the 50-cell threshold over the 3-day recovery period. Fifth, tissue toxicity from local delivery in rat muscle is designed to assess local tissue reaction to high local concentrations, not to assess systemic toxicity. The systemic data may not represent the results one would see

Table 3. Amphotericin B concentrations in serum are tabulated by time and delivery formulation

\begin{tabular}{lll}
\hline Formulation & Day 1 $(\mu \mathrm{g} / \mathrm{mL})$ & Day 3 $(\mu \mathrm{g} / \mathrm{mL})$ \\
\hline $\begin{array}{l}\text { Liposomal amphotericin B } \\
\quad 200 \mathrm{mg} / \mathrm{batch}\end{array}$ & $0.01 \pm 0.03$ & 0 \\
$\begin{array}{l}\text { Liposomal amphotericin B } \\
\quad 800 \mathrm{mg} / \mathrm{b} a t c h\end{array}$ & $0.03 \pm 0.05$ & $0.04 \pm 0.07$ \\
$\begin{array}{l}\text { Amphotericin B deoxycholate } \\
800 \mathrm{mg} / \mathrm{b} \text { atch }\end{array}$ & $0.09 \pm 0.12$ & $0.11 \pm 0.19$ \\
\hline
\end{tabular}

Values are mean $\pm \mathrm{SD}$.
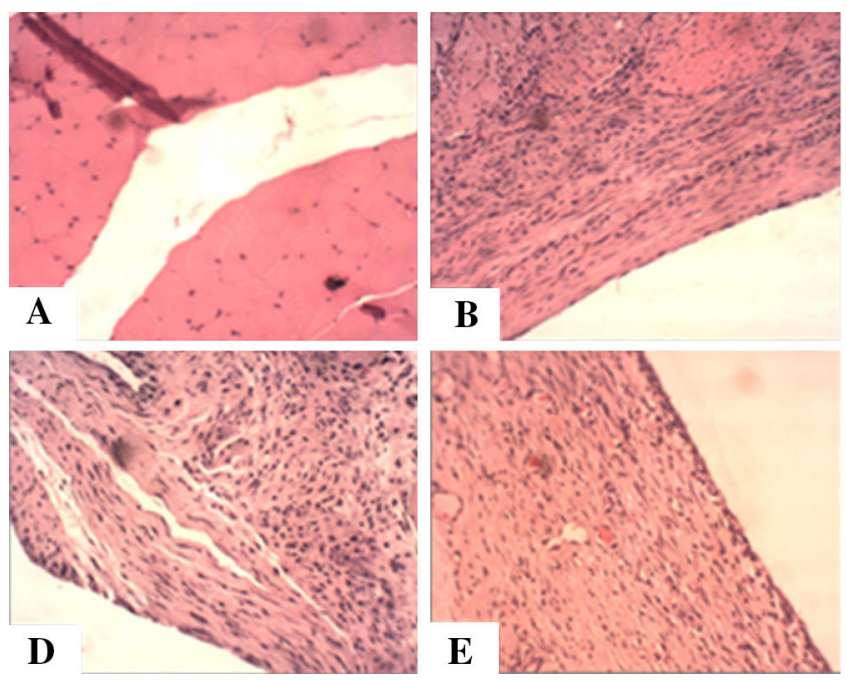

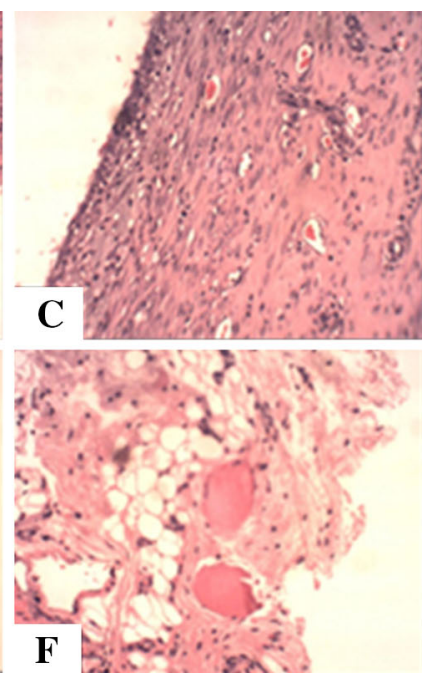

liposomal amphotericin B, (D) fibrous encapsulation around ALBC rods that contained $800 \mathrm{mg} / \mathrm{batch}$ liposomal amphotericin $\mathrm{B}$, (E) fibrous encapsulation around ALBC rods that contained $800 \mathrm{mg} / \mathrm{batch}$ amphotericin B deoxycholate, and $(\mathbf{F})$ extensive necrosis in response to the methyl cyanoacrylate-positive control injection. 
if relatively larger volumes of ALBC were used. Finally, it is difficult to scale the systemic exposure from rats to humans for controlled release of a lipophilic drug like amphotericin B. Although the systemic exposure we recorded in our study was negligible, hip or knee spacers formed from similar ALBC used in clinical practice have a much larger surface area. Considering the proportionally larger volume of distribution in humans and levels of $1.2 \mu \mathrm{g} / \mathrm{mL}$ have been reported from the first 24 hours postoperatively without signs of toxicity [11], systemic exposure is unlikely to be higher than we measured.

We found that the liposomal formulation of amphotericin B was less cytotoxic in vitro than the deoxycholate formulation. We observed little to no toxicity from 5-hour exposure to all the concentrations of the liposomal formulation that we studied. This is very different from toxicity seen with amphotericin B deoxycholate, which is consistent with previously reported data [6]. Toxicity from amphotericin B from the deoxycholate formulation occurred at lower concentrations than from the liposomal formulation. The phospholipid in the liposomal formulation of amphotericin B leads to higher release from ALBC but likely also reduces toxicity through the formation of liposomes, limiting exposure to free drug [4]. After removal of amphotericin B from the growth media, cells that were exposed to these sublethal concentrations increased their numbers over 3 days [6]. We are unaware of other reports on the local effects of liposomal amphotericin B. Harmsen et al. found that fibroblasts and osteoblasts were able to resume proliferating after exposure to 5 to $10 \mu \mathrm{g} /$ $\mathrm{mL}$ of amphotericin $\mathrm{B}$ deoxycholate for 7 days [6]. The fibroblasts we used were less resilient, being killed by exposure to amphotericin B deoxycholate of $5 \mu \mathrm{g} / \mathrm{mL}$ or greater. Cunningham et al. reported that amphotericin B release from ALBC with $800 \mathrm{mg}$ liposomal amphotericin B was active at inhibiting candida growth [4]. Clinical trials are needed to determine if locally delivered liposomal amphotericin B improves outcomes for treatment of fungal orthopaedic infections.

For the in vivo local delivery from ALBC, the primary reaction observed with all the ALBC groups, regardless of dosing or drug type, was minimal foreign body reaction with fibrous encapsulation around the implant and no additional tissue response. The encapsulation response was likely attributable, in part, to the stiff material property of the ALBC in the mobile soft tissues of the rat paraspinal muscles. We elected to assess toxicity to local release in muscle instead of bone because we felt that muscle would be more sensitive to potential toxicity from antifungal delivery, and the results could be more easily interpreted using ASTM F981-04 as a guide. There was no observed necrosis or adverse cellular response in any of the ALBC groups. Systemic concentrations of amphotericin B in our study were very low and the WBC counts were within normal reference ranges throughout the experiment, consistent with the only published clinical report in which local levels were $1.2 \mu \mathrm{g} / \mathrm{mL}$ during the first 24 hours postoperatively after local amphotericin B deoxycholate delivery from bone cement [11]. Both the low systemic levels and the lack of elevated WBC support the concept that local delivery of amphotericin B limits systemic exposure.

There is important disagreement between the in vitro and in vivo data. It is interesting that the in vivo histology provided no evidence of local toxicity occurring. This was surprising considering there is a six- to eightfold greater release for liposomal amphotericin B compared with amphotericin B deoxycholate at equal loading amounts in ALBC [4]. The absence of local toxicity in our amphotericin B deoxycholate groups may be in part the result of very low release of amphotericin $\mathrm{B}$ deoxycholate from the ALBC [4]. The lack of tissue toxicity to ALBC made with liposomal amphotericin $B$ may be the result of formation of liposomes after corelease of the amphotericin B and the phospholipid. In previous experiments with liposomal amphotericin B, we found that the drug actively inhibited fungal growth in fluid culture but did not produce zones with concentration-dependent sizes during Kirby-Bauer assays. We attributable the discrepancy to reformation of liposomes after release from the cement [4].

Our data provide evidence that, although amphotericin B can be highly toxic, both systemically and in cell culture, ALBC formulated with liposomal amphotericin B does not lead to local tissue injury in vivo. Our findings are also consistent with low systemic exposure to amphotericin B from local delivery of both the liposomal and deoxycholate formulations. Clinical studies are needed to determine the liposomal amphotericin B loads in ALBC that are tolerated clinically and what amphotericin B levels are achieved after local delivery.

Acknowledgments We thank Christine Pauken $\mathrm{PhD}$, for her assistance with the cell culture studies performed in this work.

\section{References}

1. Altman D. Practical Statistics for Medical Research. 2nd ed. Boca Raton, FL, USA: Chapman \& Hall/CRC; 2006.

2. Bekersky I, Fielding RM, Dressler DE, Lee JW, Buell DN, Walsh TJ. Pharmacokinetics, excretion, and mass balance of liposomal amphotericin B (AmBisome) and amphotericin B deoxycholate in humans. Antimicrob Agents Chemother. 2002;46:828-833.

3. Bristol Myers Squibb Canada. Fungizone Product Monograph. Available at: www.bmscanada.ca/static/products/en/pm_pdf/ Fungizone_EN_PM.pdf. Accessed January 2, 2015.

4. Cunningham B, McLaren AC, Pauken C, McLemore R. Liposomal formulation increases local delivery of amphotericin from bone cement: a pilot study. Clin Orthop Relat Res. 2012;470: 2671-2676. 
5. Fields BT, Bates JH, Abernathy RS. Amphotericin B Serum concentrations during therapy 1. Appl Microbiol. 1970;19:955-959.

6. Harmsen S, McLaren AC, Pauken C, McLemore R. Amphotericin B is cytotoxic at locally delivered concentrations. Clin Orthop Relat Res. 2011;469:3016-3021.

7. Hong Y, Ramzan I, McLachlan AJ. Hepatobiliary disposition of liposomal amphotericin B in the isolated perfused rat liver. $J$ Pharm Sci. 2005;94:169-176.

8. Kweon C, McLaren AC, Leon C, McLemore R. Amphotericin B delivery from bone cement increases with porosity but strength decreases. Clin Orthop Relat Res. 2011;469:3002-3007.

9. Loeb WF, Quimby F. Clinical Chemistry of Laboratory Animal. 2nd ed. Philadelphia, PA, USA: CRC Press; 1999.

10. Lynch AS, Robertson GT. Bacterial and fungal biofilm infections. Аппи Rev Med. 2008;59:415-428.
11. Marra F, Robbins GM, Masri BA, Duncan C, Wasan KM, Kwong $\mathrm{EH}$, Jewesson PJ. Amphotericin B-loaded bone cement to treat osteomyelitis caused by Candida albicans. Can J Surg. 2001;44:383-386.

12. Miller RB, McLaren AC, Pauken C, Clarke HD, McLemore R. Voriconazole is delivered from antifungal-loaded bone cement. Clin Orthop Relat Res. 2012;471:195-200.

13. Ramage G, Rajendran R, Sherry L, Williams C. Fungal biofilm resistance. Int J Microbiol. 2012;2012:e528521.

14. Subcommittee F04.16 on Biocompatibility Test Methods. ASTM F981-04(2010), Standard Practice for Assessment of Compatibility of Biomaterials for Surgical Implants With Respect to Effect of Materials on Muscle and Bone. 2010. Available at: www.astm.org/Standards/F981.htm. Accessed January 2, 2015. 